\title{
1 Mesenchymal Stromal Cell Delivery via an Ex Vivo Bioreactor 2 Preclinical Test System Attenuates Clot Formation for Intravascular 3 Application
}

4

Brian O’Rourke $^{1 *}$, Sunny Nguyen ${ }^{1 *}$, Arno W. Tilles ${ }^{1}$, James A. Bynum ${ }^{2}$, Andrew P Cap ${ }^{2}$, Biju Parekkadan ${ }^{1,3,4,5}$, Rita N. Barcia ${ }^{1}$

${ }^{1}$ Sentien Biotechnologies, Inc., Lexington, MA 02421 USA

2 Blood and Coagulation Research Program, U.S. Army Institute of Surgical Research, Fort Sam Houston, Texas.

${ }^{3}$ Department of Surgery, Center for Surgery, Innovation, and Bioengineering, Massachusetts General Hospital, Harvard Medical School and Shriners Hospitals for Children, Boston, Massachusetts 02114, USA

${ }^{4}$ Harvard Stem Cell Institute, Cambridge, Massachusetts 02138, USA

${ }^{5}$ Department of Biomedical Engineering, Rutgers University, Piscataway, New Jersey 08854, USA

* Equal contribution

Corresponding author: rita.barcia@sentienbiotech.com

Key words: Mesenchymal stromal cells, ex vivo, clotting, heparin, clot formation time, perfusion, bioreactor, hypercoagulable, COVID-19, IBMR, instant blood-mediated inflammatory reaction

\section{Author Contributions}

Conceptualization, BOR, SN, AT, AC, JB, BP, RNB. Execution of Experiments, BOR, SN; Data Analysis and Review, BOR, SN, AT, AC, JB, RNB; Manuscript Preparation, BOR, SN, AT, AC, JB, BP, RNB; Funding Acquisition, BP, RNB. 
O’Rourke et al, Bioreactor Attenuates Clot Formation

\section{Abstract}

36

37

While mesenchymal stromal cells (MSCs) are an appealing therapeutic option for a range of clinical applications, their potential to induce clotting when used systemically remains a safety concern, particularly in hypercoagulable conditions, such as in patients with severe COVID-19, trauma, or cancers. Here, we tested a novel ex vivo approach aimed at improving the safety of MSC systemic administration by use of a bioreactor. In this device, MSCs are seeded on the outside of a hollow-fiber filter, sequestering them behind a hemocompatible membrane, while still maintaining cross talk with blood cells and circulating signaling molecules. The potential for these bioreactor MSCs to induce clots in coagulable plasma was compared against "free" MSCs, as a model of systemic administration, which were directly injected into the circuit. Our results showed that physical isolation of the MSCs via a bioreactor extends the time necessary for clot formation to occur when compared to "free" MSCs. Measurement of cell surface data indicates the presence of known clot inducing factors, namely tissue factor and phosphatidylserine. Results also showed that recovering cells and flushing the bioreactor prior to use further prolonged clot formation time. Further, application of this technology in two in vivo models did not require additional heparin to maintain target ACT levels relative to the acellular device. Taken together, the use of hollow fiber filters to house MSCs, if adopted clinically, could offer a novel method to control systemic MSC exposure and prolong clot formation time.

\section{Introduction}

Mesenchymal stromal cells (MSCs) are potent immunoregulators with strong preclinical data that support their application in a wide range of clinical conditions [1-4]. MSCs can provide therapeutic benefit to patients suffering from systemic inflammation by effectively immunomodulating peripheral blood cells to reduce inflammatory signaling and promote homeostasis. Significant evidence of these MSC derived effects have been shown in vitro, in animal models, and in clinical trials, including recently 
under an emergency IND application or expanded access protocol with COVID-19 infected patients [5-

59 10]. However, it is known that under certain conditions MSCs may be pro-coagulable and promote instant blood-mediated inflammatory reaction (IBMIR), likely through the expression of known coagulation factors on their cell surfaces and in the production of cellular microvesicles [11-14]. Two such factors, tissue factor and phosphatidylserine are known to be integral in physiological coagulation [15-18]. Phosphatidylserine is a major component of cell-based coagulation, enhancing coagulation activity through the charge-based binding of coagulation factor zymogens and cofactors to increase formation of the tenase and prothrombinase complexes [19-21]. This binding effectively enhances clot formation potential, exacerbating a response when a physiological trigger of coagulation, such as tissue

67 factor, is present $[18,21,22]$. When MSCs are introduced into systemic circulation they bring with them the tissue factor expressed on their cell surface. MSCs sourced from different donors and locations

69 (bone marrow, BM-MSCs, adipose-derived, AD-MSCs, umbilical cord, UC-MSCs) vary in tissue factor expression, with higher levels of tissue factor correlating with quicker clot formation and IBMR. BM-

71 MSCs, the most commonly used, have been widely shown to have the least tissue factor expression [16, $7218]$. groups over the last decade. Advances have been made to mitigate these risks including changing the MSC mode of delivery away from systemic exposure via intravenous (IV) infusion towards localized injection. However, in some cases, systemic IV infusions could provide the highest therapeutic potential.

77 Minimizing clot formation potential could improve therapeutic efficacy, and also increase safety of the

78 treatment in hypercoagulable patients such as patients with COVID-19 in the ICU [23-26]. To that end,

79 increasingly rigorous release criteria during MSC clinical manufacturing are now being applied. Cell 
82

evaluated, including shifting delivery of the MSCs from intravenous to intramuscular administration, emphasizing higher cell viability, or even using gene modification to promote cell survival [28-31].

We propose an alternative approach which would minimize direct exposure of the MSCs to blood and contain MSCs in one location. We utilized a recently described perfusion platform that incorporates a hollow-fiber filter into a fluid circuit to compartmentalize the MSCs, while still allowing exchange of signaling molecules from perfusate to cells, and vice-versa [32]. MSCs within this platform were shown to effectively retain their immunomodulatory capacity and alter perfused lymphocyte proliferation, activation, and cytokine production in an MSC dose and duration exposure dependent manner, despite having minimal direct contact with the blood cells.

Benchtop coagulation assays, including microfluidic setups, are becoming increasingly translationally relevant[33]. Here, we used a MSC bioreactor platform to assay whether limiting the direct exposure of fresh frozen pooled plasma from healthy patients to MSCs, and therefore the available tissue factor and phosphatidylserine of the MSCs, would affect clot formation time (CFT) in a modified plasma-based clot formation assay [34, 35]. Plasma was perfused through bioreactors seeded with MSCs as well as through circuits in which cells were directly injected into the perfused plasma to make comparative CFT measurements. We were able to show that bioreactor use significantly prolonged CFT relative to direct injection of the MSCs. Flushing of concentrated soluble factors from bioreactors further contributed to prolonged CFTs. Lastly, the previously proposed clinical solution for MSC driven clot formation, anticoagulation with heparin [36-39], was shown to be effective in both perfusion setups. These results suggest this new modality for systemic MSC delivery may offer a safer alternative to intravenous MSC injection. The clinically scaled ex vivo engineered MSC delivery is currently undergoing testing in a Phase I/II trial in acute kidney injury (AKI) and COVID-19 associated AKI. 
O’Rourke et al, Bioreactor Attenuates Clot Formation

\section{Materials and Methods}

\section{MSC Cell Source and Culture Processes}

107 Human bone marrow derived mesenchymal stromal cells were isolated from 3 separate donors. Cells 108 were cultured and propagated either in-house using proprietary techniques at developed Sentien 109 Biotechnologies (MA, USA) or RoosterBio (MD, USA) and cryopreserved at early passage (P3-P5). Cells 110 were cultured using 2D planar growth conditions. Cells from Sentien Biotechnologies were cultured in 111 aMEM media (HyClone, UT, USA, SH3A5195) containing FGF and FBS, while Roosterbio cells were 112 expanded in xeno-free RoosterNourish-MSC-CC media (RoosterBio, MD, USA, KT-021). All human 113 samples were obtained from commercial vendors under a consented protocol for research purposes 114 only.

$\underline{\text { MSC Direct Injection }}$

116 MSCs were thawed from cryopreservation into citrated fresh-frozen pooled plasma (FFPP) (George King

117 Bio-Medical, KS, USA) and counted via Trypan Blue exclusion. Each direct injection subgroup was

118 processed individually and subgroups were never combined. The fresh thawed direct injection subgroup 119 was placed into plasma after counting and directly injected for perfusion (Figure 2). The recovered direct 120 injection subgroup allowed for 24 hours of recovery on a cell culture dish, followed by dissociation with 121 TryPLE, counting via Trypan Blue exclusion, and placing into plasma prior to direct injection. Cells for use 122 in washed groups were washed with saline directly after thaw, pelleted and resuspended in fresh 123 citrated media prior to direct injection. 
O’Rourke et al, Bioreactor Attenuates Clot Formation MSCs were thawed from cryopreservation into aMEM supplemented with $10 \%$ HSA and counted via Trypan Blue exclusion prior to seeding in device. The desired cell number was suspended into $9 \mathrm{~mL}$ of aMEM and then seeded into saline-primed microreactors (Spectrum Laboratories, CA, USA; C02-P20U05) with 0,1 , or $3 \times 10^{6}$ viable cells per device $(0 \mathrm{M}, 1 \mathrm{M}, 3 \mathrm{M}$, respectively). Excess media flowed through the semi-permeable hollow fibers while cells remained within the extraluminal space of the reactor. Microreactors used were $20 \mathrm{~cm}$ long with an internal surface area of $28 \mathrm{~cm}^{2}$. Within each microreactor are nine $0.5 \mathrm{~mm}$ diameter fibers comprised of polyethersulfone with a $0.2 \mu \mathrm{m}$ pore size. The total internal volume of the microreactor is $1.5 \mathrm{~mL}$.

Depending on the group, microreactors were either used immediately or incubated at $37^{\circ} \mathrm{C}$ for 2 hours to allow for cell attachment and were subsequently held for 24 hours at room temperature prior to integration into the circuit. This hold time intends to mimic the time between device manufacture and potential clinical application.

Following hold, select MRs were subjected to flushing. Prior to connection to the perfusion circuit sterile saline $(4.5 \mathrm{~mL})$ was pushed via syringe through the extracapillary port of the MR. Discharge exited through the intracapillary port.. Samples from the extracapillary space were collected prior to and post flush to measure soluble levels of phosphatidylserine and tissue factor.

Large scale bioreactors were used in the animal studies. Similar to the microreactor fill/finish process, following MSC thaw, cell and media suspension was perfused through the extracapillary port onto semipermeable hollow fibers (Asahi Kasei Medical Inc, IL, USA, OP-05W(A). Cells remained within the extraluminal space of the reactor while excess media perfused through the membranes out the intracapillary port. Thawed vials used in these assays were comprised of cells with a minimum $80 \%$ viability. Bioreactors were seeded with either 0,250 , or $750 \times 10^{6}$ viable cells per unit. 
O’Rourke et al, Bioreactor Attenuates Clot Formation

149

150

151

152

153

154

155

156

157

\section{Fresh-Frozen Pooled Plasma}

Fresh-Frozen Pooled Plasma was collected and citrated via FDA licensed blood centers from prescreened healthy donors (Geroge King Bio-Medical, KS, USA). No buffers or stabilizers were added. Plasma was frozen within 30 minutes of collection at $-70^{\circ} \mathrm{C}$ from a pool of $>50$ donors per lot. This plasma still contains many essential factors for clot initiation, including prothrombin which can be activated with the addition of calcium via $\mathrm{CaCl} 2$ to form firm clots over time. Testing was done to ensure normal values for PT, aPTT, fibrinogen, dRVVT normalized ratio, Factors II, V, VII, VIII, IX, X, XI, and XII.

\section{$\underline{\text { Plasma Perfusion }}$}

After thawing $5 \mathrm{~mL}$ of FFPP via water bath $\left(37^{\circ} \mathrm{C}\right), 50 \mathrm{uL}$ of $1 \mathrm{M} \mathrm{CaCl}_{2}$ was added to the plasma within a capped syringe and inverted. The plasma was loaded into prepared perfusion circuits with $(0,1$, and $\left.3 \times 10^{6} \mathrm{MSCs}\right)$ and without microreactors via the syringe port and perfused at a flow rate of $1 \mathrm{~mL} / \mathrm{min}$ for 5 minutes. Plasma was then extracted from the circuit via the syringe port, aliquoted into microplate wells and placed within the spectrophotometer (Synergy Mx, BioTek) for reading at $405 \mathrm{~nm}$ every 10 seconds for a total of 45 minutes.

Positive controls of Innovin (Siemens Healthcare Diagnostics, Germany) or Factor IXa (Haematologic Technologies, VT, USA) were used separately, where designated, at 1:50,000. These positive controls were added prior to $\mathrm{CaCl}_{2}$ addition to ensure equal mixing before coagulation initiation. $1 \mathrm{M} \mathrm{CaCl}_{2}$ was added at 1:100.

Unfractionated heparin (Grifols, Spain) was used at $1.5 \mathrm{U} / \mathrm{mL}$ in the designated groups. Heparin was added prior to $\mathrm{CaCl}_{2}$ addition to ensure equal mixing before coagulation initiation. 
O’Rourke et al, Bioreactor Attenuates Clot Formation

171

172

173

174

175

176 177 added to the time required to obtain the $1 / 2$ maximal spectrophotometric value, as determined using the 178 clot formation time formula. Combined, these measurements capture the clot formation time.

179

180

181

182

183

184

185

186

187

188

189

190

191

\section{Clot Formation Time Analysis and Graphing}

Clot formation time measurements incorporated the sum of two time periods. The first period initiates when plasma has been recalcified through the addition of $\mathrm{CaCl}_{2}$ and continues through perfusion until transfer of the samples into microwells for analysis on the spectrophotometer. This value is immediately recorded by the operator. The second time period occurs when the spectrophotometric readings begin and continues for 45 minutes. At the completion of the study the elapsed time in the first period is

Resulting values were then graphed and statistically analyzed using unpair student's t-test (GraphPad Software, La Jolla, CA). Results are presented as mean \pm standard deviation. Values of $p<0.05$ were considered statistically significant for all analyses.

\section{Flow cytometry}

To measure tissue factor by flow cytometry, staining was done using CD142-APC monoclonal antibody (eBiosciences) in a total volume of $100 \mu \mathrm{L}$ Stain Buffer containing FBS and $\leq 0.09 \%$ sodium azide (BD

Biosciences). Samples were incubated for $15 \mathrm{~min}$ at $4^{\circ} \mathrm{C}$ and analyzed on a FACSCanto II flow cytometer (BD Biosciences) using BD FACSDiva v6.1.1 software. Mouse PE IgG1 kappa isotype antibody (eBioscience) was used as a negative control.

Annexin V staining was done using the FITC Annexin V Apoptosis Detection Kit (BioLegend) according to the manufacturer's instructions. Fresh thawed MSCs were used to optimize fluorescence compensation.

\footnotetext{
Flow cytometry analysis was performed in FlowJo (FlowJo LCC, OR, USA; version 10.7).
} 
O’Rourke et al, Bioreactor Attenuates Clot Formation

\section{Mongrel Dog Perfusion}

Eighteen male mongrel dogs were randomized onto the study and underwent surgery for placement of a dialysis catheter (Toxikon Corp, Beford MA). Animals received buprenorphine $(0.01 \mathrm{mg} / \mathrm{kg}$, IM) presurgery, PM the day of surgery and AM the day after surgery, and cefazolin $(22 \mathrm{mg} / \mathrm{kg}$, IV/IM) presurgery, then daily for two additional days post-surgery. After a 2 day wash out period, animals were dosed according to their group assignment with 6 animals assigned to either a 0M, 250M, or 750M MSC dose group. Heparin was administered throughout treatment with all animals first receiving a bolus of heparin at $150 \mathrm{U} / \mathrm{kg}$ and then a continuous infusion of $25 \mathrm{U} / \mathrm{kg}$ every hour. All animals underwent a 24hour perfusion (+/- 1 hour), with the exception of animal 1003 (Group 1, Control) which was stopped after 22 hours due to low blood flow rate through the catheter.

\section{Porcine Acute Myocardial Infarction Model Perfusion}

On Day 0, 8 Yorkshire pigs underwent induced myocardial infarction of the anterior/septal left ventricle by 45-minute occlusion of the left anterior descending artery (CBSet Inc., Lexington, MA). After one hour of reperfusion/stabilization, animals were connected to the extracorporeal loop via the jugular vein which enabled whole blood circulation through a large scale bioreactor for a period of up to 12 hours. Bioreactors were seeded with either 0 or 750 million human bone-marrow derived MSCs, 24 hours prior to perfusion ( $n=4$ per group). Heparin was administered throughout the treatment, first as a bolus of $225 \mathrm{U} / \mathrm{kg}$ and then intermittently to ensure ACTs remained above 300 seconds. Serum troponin levels were assessed at baseline 12 and 24 hours after infarction induction. At 72 hours post injury induction animals were sacrificed with their hearts excised and dyed with Evans Blue and 1\% TCC. Infarct area was determined through tracing of digitized images of section via the morphometric software system Olympus cellSens (Version 1.17). 
O’Rourke et al, Bioreactor Attenuates Clot Formation

\section{Results}

218 Development of an assay to test CFT under perfusion

219 We developed an approach in which we could test human plasma compatibility of allogeneic MSCs

220 across multiple extents of cell exposure. Cryopreserved MSCs could either be injected into the plasma

221 directly after thaw (Fig. 1A), cultured for 24 hours after recovery and then directly injected into plasma

222 (Fig. 1B), or seeded into hollow-fiber microreactors with a semi-permeable membrane, allowed to

223 attach, and held for up to 24 hours before being subjected to perfusion (Fig. 1C). These ranges of

224 administration broadly represent many of the systemic administration options available today and allow

225 the comparison of varying degrees of MSC to plasma exposure and the effects of MSC culture

226 conditions.

227 After perfusion, plasma was collected from the circuit via the syringe port and placed into microwells for

228 spectrophotometric reading over time. The point at which a clot was formed was determined by using

229 the resultant spectrophotometric readout and formula (Fig. 1D, E) to calculate the $1 / 2$ maximal value, a

230 point previously determined to designate clot formation [34, 35]. Higher values for clot formation times

231 indicate slower clot formation within the plasma.

234 Consistent with previous work [12], our fresh-frozen pooled plasma-based (FFPP) clot formation assay

235 showed that the presence of MSCs accelerated clot formation under flow conditions relative to acellular

236 controls (Figure 2). Interestingly, the same experimental setup run with plasma isolated from an 
individual donor 24 hours after collection instead of FFPP did not result in significantly different clotting times between cellular and acellular groups, likely as it wasn't frozen directly after being pulled (Figure occurred within the microwell via increases in measured OD over time (Figure 2A). Results from our

241 assays showed that the direct injection (DI) of freshly thawed MSCs into the plasma flow circuit

242 significantly hastened the onset of clot formation when compared to circuits using bioreactor housed

243 MSCs. Both the 1M BM-MSC and 3M BM-MSC direct injection groups induced clot formation during the

244 initial perfusion, prior to spectrophotometric reading. All other groups completed perfusion and

245 spectrophotometric reading (Figure 2B). These findings were consistent across 3 separate MSC donors

246 (Figure S2).

247 Furthermore, MSC dose played a role in clot formation. Increases in the number of cells administered

248 were significantly associated with shorter CFTs across both administration routes. However, when

249 comparing similarly seeded MRs to direct injection with the same number of cells, CFT was significantly

250 slower in the groups where MSCs were housed in the bioreactor (Figure 2B).

Immobilizing MSCs in the bioreactor allows for cell recovery post-thaw, a process proposed to reduce

254 the surface exposure of pro-coagulation factors [27]. In order to investigate the effect of recovering

255 MSCs on clot formation, two known pro-coagulant factors- tissue factor and phosphatidylserine - were

256 measured prior to perfusion. Flow cytometric analysis of the surface markers on both the freshly thawed

257 and MSCs allowed to recover for 24 hours in culture, showed that cell recovery had significantly lowered 258 the levels of phosphatidylserine and tissue factor (Figure 3A). 
We next asked whether recovering MSCs post thaw had an effect on clot formation. CFTs were compared between freshly thawed cells (unwashed or washed) and cells recovered for 24 hours. Both unwashed and washed freshly thawed conditions quickly clotted at similar times during perfusion suggesting that washing to remove debris and cryopreservative did not affect clot time. However, CFT was significantly prolonged by allowing for 24 hours of recovery in culture prior to injection (Figure 3B). limiting the direct interaction of MSC surface markers and plasma could reduce the rate of clot formation. Interestingly, recovery of MSCs within a microreactor did not significantly affect measured

267 CFTs relative to fully recovered MSCs (Figure 3B). MSCs that were thawed, seeded into MRs, and

268 immediately perfused, clotted at the same time as MSCs that were seeded and allowed to recover for 24

269 hours. These results suggest that though recovery of cells post thaw significantly reduces MSC induced

270 clotting, housing them in an adherent state on the outside of a hollow fiber seems to further, and more

271 significantly, reduce their clotting potential even without any recovery period.

273 Removing soluble factors from the MSC reactor prolongs CFT

274 While seeding MSCs on the hollow fiber membrane resulted in prolonged CFT, cellular contribution was

275 still observed as all cellular microreactor circuits clotted in a dose dependent manner (Figure 2B). While

276 in the 24-hour hold period post-attachment, it is likely that MSC derived factors accumulate within the

277 microreactor and may contribute towards clotting. To directly assay this, we integrated a saline flush of

278 the microreactor into our protocol. After the 24-hour hold and just prior to perfusion, MRs were flushed

279 with $3 X$ column volume $(4.5 \mathrm{~mL})$ of saline. Samples from the microreactor were collected pre-and post-

280 flush and subjected to flow cytometric analysis for measurement of tissue factor and

281 phosphatidylserine. Pre-flush samples showed higher levels of phosphatidylserine and tissue factor in 
282 the cellular group as expected. Post-flush samples showed clear reductions in both factors,

283 demonstrating that soluble factors can be effectively flushed out of the hollow fiber filter (Figure 4A).

284 Flushing the microreactors significantly prolonged CFT in higher doses (3M), while in lower doses (1M)

285 significance could not be reached. (Figure 4B). These data indicate that soluble factors (e.g.

286 phosphotidylserine) are present in the extracapillary space of the bioreactor and can accumulate to

287 contribute to accelerated clot formation.

$\underline{\text { Heparin administration prevents MSC induced clotting in vitro }}$

Despite significantly reducing the clotting potential of MSCs, bioreactors loaded with the cells at higher

291 doses (3M) still induced earlier clotting when compared to acellular or low-dose (1M) cell bioreactors.

292 Plasma spiked with heparin was used to investigate the potential efficacy of administered

293 anticoagulation in preventing clot formation within the circuitry. Administration of $1.5 \mathrm{U} / \mathrm{mL}$ of heparin

294 across all groups was able to completely prevent clot formation, even in the presence of positive control

295 Innovin or 3M directly injected MSCs (Figure 5).

\section{$\underline{\text { Heparin prevents MSC induced clotting in vivo }}$}

299 The bioreactor setup can be scaled up with larger filters to allow for perfusion in large animal models.

300 Previous work in in vivo models showed that heparin administration could effectively reduce

301 procoagulant activity of MSCs [35]. To reduce the number of animals used, here we compared only

302 between bioreactor groups, no direct injection animal studies were conducted. We first tested feasibility

303 of perfusion of the device in vivo in a healthy canine model. Animals were all heparinized to assure

304 safety as extracorporeal treatments (even without cells) have intrinsic clotting potential. Dogs were 
grouped into cohorts based on the number of MSCs loaded into a scaled up bioreactor, with doses of 0 million, 250 million, and 750 million ( $n=6$ dogs per group) and perfused for 24 hours. No clotting was seen in any group (data not shown).

Next, we asked the question of whether clotting in vivo would be observed under an pathological conditions, such as acute organ failure, where systemic inflammation may perturb the coagulation pathways. For this purpose a porcine animal model of acute myocardial infarction (AMI) was used

311 (Figure 6 A). AMI was induced, animals were re-perfused/stabilized for 1 hour and then connected to 312 the bioreactor perfusion circuit for 12 hours. All animals were perfused without events for 12 hours,

313 with each group showing cardiac injury biomarker induction (Figure 6 B) and similar infarct size (Figure 6

314 C). Heparin was administered throughout the perfusion process to maintain a minimum activated

315 clotting time (ACT) of at least 300 seconds (as mandated by IACUC), with neither group requiring

316 significantly more heparin than the other (Figure 6 D, E). These data support the use of MSC bioreactors 317 without additional heparin requirements beyond what is used in acellular extracorporeal treatments.

\section{Discussion}

In the absence of clear clinical benefit of early allogeneic MSC human trials to meet their therapeutic

321 endpoints, there has been a major focus in recent years to improve the reliability and consistency of the 322 therapeutic cells delivered [40]. Improvements in manufacturing processes, more stringent release 323 testing and biobanking has provided a reproducibility to the cell production that has contributed to a 324 clinically approved therapy [8, 28, 41-43]. However, many cellular concerns still exist, including handling 325 at point of care, thawing, route of delivery, hemocompatibility, and dosing. Our studies here focused on comparing the potential risks of one of those concerns, MSC induced coagulation, between direct

327 infusion and a modified ex vivo, systemic approach. 
Most commonly, allogeneic MSCs are delivered for therapeutic effect through systemic administration

329 (intravenous or intra-arterial) accounting for about half of all published studies [28]. Systemic

330 introduction has been described as the least invasive, most reproducible, and provides the MSCs the

331 most direct access to modulate systemic inflammation [44]. However, this route of administration may

332 increase risks to certain hypercoagulable patients given that MSCs are known to express coagulation

333 factors both on their cell surface and on the exosomes and vesicles they secrete, namely tissue factor

334 and phosphatidylserine $[16,41,45]$. Further, systemically introduced MSCs can rapidly get trapped in

335 the lungs or be cleared, reducing their potential efficacy [40]. Because of these concerns, where possible

336 and concordant with the mechanism of action (MoA), alternatives to systemic administration are

337 increasingly utilized, including intramuscular infusions, topical, direct tissue injections, and intracoronary

338 delivery. While useful for localized applications including tissue regeneration, these routes of delivery

339 are not used to treat systemic applications such as GvHD, and present limitations of their own in terms

340 of feasibility, reproducibility, and efficacy $[46,47]$.

341 Here, in concert with the previously mentioned improvements with cellular production, we assayed the

342 value of incorporating an experimental setup which confines MSCs behind the membrane of a hollow-

343 fiber filter. Given that much of the MSCs' ability to induce clot formation arises from its cell surface

344 markers and secreted vesicles, we considered that the confinement of the cells and their procoagulant

345 expressing surface markers in one extraluminal location may reduce the rate at which clot formation

346 occurs. We used an existing bioreactor platform known to retain MSC immunomodulatory capacity in

347 combination with modifications to an existing clot formation assay to assess cellular effect on CFT in this

348 immobilized state relative to direct injection [32, 34, 35]. Through this platform we perfused citrated,

349 platelet poor fresh-frozen pooled plasma. This plasma contains many essential factors for clot initiation,

350 including prothrombin which can be activated with the addition of calcium via $\mathrm{CaCl}_{2}$ to form firm clots

351 over time. 
352 Immediately after $\mathrm{CaCl}_{2}$ addition, this coagulable (but still liquid) plasma is perfused through the hollow-

353 fiber filter platform. As expected, direct injection of MSCs into the coagulable plasma perfusion circuit

354 led to rapid clot formation in a dose-dependent manner. Interestingly, perfusion of coagulable plasma

355 through bioreactors seeded with MSCs resulted in clotting at rates significantly slower than their

356 comparable direct injection groups, suggesting that free, circulating MSCs increase thrombosis risk more

357 than bioreactor immobilized MSCs. Like the direct injection group, the MSC dose seeded in the

358 bioreactor was predictive of CFT with higher doses inducing quicker clots, likely through the increased

359 production of pro-coagulable MSC factors. It is also important to note that the presence of a filter

360 (acellular microreactor) in the circuit induced clotting faster than a circuit without a microreactor

361 supporting the idea that high surface area biomaterials increase factor adsorption and may contribute

362 to expedited clotting [48]. Alternative approaches such as heparin coating the hollow fiber filters prior

363 to MSC seeding may reduce surface adsorption and adhesion, lowering this inherent clot induction

364 potential [49-51].

365 Historically, failed MSCs trials have been in part attributed to poor cell processing, including delivery of

366 dead and/or coagulable cells $[40,43,52,53]$. In this study, washing of cells post thaw did not affect CFT

367 significantly. However, recovery of cells for 24 hours post thaw did reduce clot formation potential. This

368 was shown to correlate with surface marker expression of tissue factor and phosphatidylserine. Both

369 decreased following recovery, correlating with a slower clot formation time relative to MSCs directly

370 injected into the circuit post thaw. Recovery culture of cryopreserved MSCs within a microreactor did

371 not have a significant impact on CFT relative to freshly thawed cells within microreactors, suggesting

372 that the MSC confinement by the hollow fiber membrane may actually be playing a role in prolonging

373 CFT. While MSCs located behind the membrane are still able to exchange their immunomodulatory

374 secreted factors with perfusing solutions, they may be sharing less of their cell surface area and may aid 
O’Rourke et al, Bioreactor Attenuates Clot Formation

375 in confining their cellular debris to the extracapillary space of the microreactor. Future studies with

376 more restrictive filter sizes may even further limit MSC exposure and further prolong CFT.

377 Having shown that cell presence shortens clot formation time we sought to use the platform to mitigate

378 that effect as much as possible. Since our microreactor is composed of hollow fibers, exchange does

379 happen through the $0.2 \mu \mathrm{m}$ pores on the fibers in the microreactor. During hold, MSCs continue to

380 produce materials and some of this accumulated material could be contributing to clot formation. To

381 assess this, we developed a flush protocol and measured steep drops in known coagulation markers.

382 Consequently, flushing resulted in slower CFTs at higher MSC doses. Future studies will assay whether

383 flushing also affects immunomodulatory potential relative to unflushed reactors [32].

384 Despite the microreactors measured effect of prolonging CFT, it did not completely abrogate the cellular

385 contribution to shortened CFT. In clinical setting anticoagulation protocols will likely be integrated to

386 ensure designated perfusion times are met. Our in vitro experiments and in vivo canine studies showed

387 that heparin administration could effectively prevent any cellular induced clot formation during

388 perfusion. However, many of the patients suffering from systemic inflammation, including those with

389 COVID-19, present with hypercoagulable plasma that will require anticoagulation prior to MSC

390 administration. Our pig model represented a more physiologically relevant condition in which inflamed

391 animals were perfused with a device scaled for human use. Under these acute injury conditions, no

392 clotting was observed in animals perfused with devices loaded with 750M MSCs and for 12 hours. The

393 lack of additional heparin requirement suggests that patients set to undergo MSC-bioreactor perfusion

394 may not need more heparin than a sham control undergoing the same procedure. Future studies

395 comparing direct infusion of MSCs to bioreactor housed MSCs would be useful to evaluate both for

396 coagulation and efficacy responses. 
O'Rourke et al, Bioreactor Attenuates Clot Formation

397

398

399

400

401

402

403

404

405

406

407

408

409

410

411

412

413

414

415

416

417

418

419

Given the slower CFT in the microreactor groups relative to the direct injection groups, it is possible that a lower dose of heparin could be administered to the microreactor groups. Future dose testing will be required to verify this. Such a finding would be clinically relevant, as reduction in the amount of heparin required to be delivered to critically ill patients may help prevent unintended health consequences.

Further, patients which are medically restricted from systemic heparin administration for risk of internal bleeding could potentially be anticoagulated regionally with citrate. Citrate could be introduced and equilibrated within the MSC bioreactor circuit, allowing MSC factors to be released but without exposing patients to the anticoagulant [54].

In the unfortunate circumstances of the COVID-19 pandemic, interest in MSC based therapies has increased markedly. Case reports, first from China and then worldwide, showed promising improvements in patient health following intravenous MSC infusion, even in severely ill patients [10, 55, 56]. While larger studies are now needed to more completely support these findings, it is clear that intravenous infusion of MSCs for systemic inflammatory conditions such as COVID-19 infection or GvHD continues to have therapeutic potential. Remestemcel-L, an ex-vivo culture-expanded adult human MSC suspension for intravenous infusion, which has received positive recommendations from the FDA for steroid-refractory acute graft-versus-host disease in pediatric patients based on an open-label study compared to historical controls [57]. The novel delivery approach described here could potentially reduce risk of clot formation from IV administered MSCs, making treatment potentially safer and more controlled than direct infusion.

\section{Conclusion}

We conclude that immobilization of MSCs in a hollow fiber filter contributes to a reduced clot initiation potential relative to directly injected MSCs. Further removal of cellular byproducts through saline flushing of the bioreactor further reduces the MSC based clot formation potential. Additional heparin 
420 does not appear to be required to maintain a designated ACT value relative to acellular perfusion

421 circuits. Taken together, combined integration of these approaches may make MSC therapies which

422 require systemic MSC exposure at less risk for coagulation-related events for a larger population,

423 including the hypercoagulable.

\section{Disclosures of Potential Conflicts of Interest}

426 The opinions or assertions contained herein are the private views of the author and are not to

427 be construed as official or as reflecting the views of the Department of the Army or the

428 Department of Defense.

$429 \mathrm{JB}$ and AC are United States government employees with no financial disclosures relevant to 430 this publication.

431 BOR, SN, AT, RNB are employees and equity shareholders of Sentien Biotechnologies. BP is

432 an equity shareholder and inventor of the technology with licensed patents to Sentien for

433 commercialization.

$434 \quad$ Funding

435 This research was conducted with private funding.

437 Data Availability Statement

438 The data that support the findings of this study are available from the corresponding author 439 upon reasonable request. 
O’Rourke et al, Bioreactor Attenuates Clot Formation

\section{References}

[1] Liu L, Wong CW, Han M, Farhoodi HP, Liu G, Liu Y, et al. Meta-analysis of preclinical studies of mesenchymal stromal cells to treat rheumatoid arthritis. EBioMedicine 2019;47:563-77.

[2] Beegle JR, Magner NL, Kalomoiris S, Harding A, Zhou P, Nacey C, et al. Preclinical evaluation of mesenchymal stem cells overexpressing VEGF to treat critical limb ischemia. Molecular therapy Methods \& clinical development 2016;3:16053.

[3] Huang YZ, Gou M, Da LC, Zhang WQ, Xie HQ. Mesenchymal Stem Cells for Chronic Wound Healing: Current Status of Preclinical and Clinical Studies. Tissue engineering Part B, Reviews 2020.

[4] Torres Crigna A, Daniele C, Gamez C, Medina Balbuena S, Pastene DO, Nardozi D, et al. Stem/Stromal Cells for Treatment of Kidney Injuries With Focus on Preclinical Models. Frontiers in medicine 2018;5:179.

[5] Parekkadan B, Milwid JM. Mesenchymal stem cells as therapeutics. Annual review of biomedical engineering 2010;12:87-117.

[6] Chamberlain G, Fox J, Ashton B, Middleton J. Concise review: mesenchymal stem cells: their phenotype, differentiation capacity, immunological features, and potential for homing. Stem cells (Dayton, Ohio) 2007;25:2739-49.

[7] Uccelli A, Moretta L, Pistoia V. Mesenchymal stem cells in health and disease. Nature reviews Immunology 2008;8:726-36.

[8] Hoogduijn MJ, Lombardo E. Mesenchymal Stromal Cells Anno 2019: Dawn of the Therapeutic Era? Concise Review. Stem cells translational medicine 2019;8:1126-34.

[9] Sánchez-Guijo F, García-Arranz M, López-Parra M, Monedero P, Mata-Martínez C, Santos A, et al. Adipose-derived mesenchymal stromal cells for the treatment of patients with severe SARS-CoV-2 pneumonia requiring mechanical ventilation. A proof of concept study: EClinicalMedicine. $2020 \mathrm{Jul}$ 10:100454. doi: 10.1016/j.eclinm.2020.100454.

[10] M T. Mesoblast's Stem Cell Therapy Shows 83\% Survival in Ventilator-Dependent COVID-19 Patients. BioSpace2020.

[11] Coppin L, Sokal E, Stéphenne X. Thrombogenic Risk Induced by Intravascular Mesenchymal Stem Cell Therapy: Current Status and Future Perspectives. Cells 2019;8.

[12] Silachev DN, Goryunov KV, Shpilyuk MA, Beznoschenko OS, Morozova NY, Kraevaya EE, et al. Effect of MSCs and MSC-Derived Extracellular Vesicles on Human Blood Coagulation. Cells 2019;8.

[13] Jung JW, Kwon M, Choi JC, Shin JW, Park IW, Choi BW, et al. Familial occurrence of pulmonary embolism after intravenous, adipose tissue-derived stem cell therapy. Yonsei medical journal 2013;54:1293-6.

[14] Moll G, Rasmusson-Duprez I, von Bahr L, Connolly-Andersen AM, Elgue G, Funke L, et al. Are therapeutic human mesenchymal stromal cells compatible with human blood? Stem cells (Dayton, Ohio) 2012;30:1565-74.

[15] Mackman N. The role of tissue factor and factor VIla in hemostasis. Anesthesia and analgesia 2009;108:1447-52.

[16] Christy BA, Herzig MC, Montgomery RK, Delavan C, Bynum JA, Reddoch KM, et al. Procoagulant activity of human mesenchymal stem cells. The journal of trauma and acute care surgery 2017;83:S164s9.

[17] George MJ, Prabhakara K, Toledano-Furman NE, Wang YW, Gill BS, Wade CE, et al. Clinical Cellular Therapeutics Accelerate Clot Formation. Stem cells translational medicine 2018;7:731-9.

[18] Chance TC, Rathbone CR, Kamucheka RM, Peltier GC, Cap AP, Bynum JA. The effects of cell type and culture condition on the procoagulant activity of human mesenchymal stromal cell-derived extracellular vesicles. The journal of trauma and acute care surgery 2019;87:S74-s82. 
O’Rourke et al, Bioreactor Attenuates Clot Formation

[19] Lentz BR. Exposure of platelet membrane phosphatidylserine regulates blood coagulation. Progress in lipid research 2003;42:423-38.

[20] Israels SJ, Rand ML, Michelson AD. Neonatal platelet function. Seminars in thrombosis and hemostasis 2003;29:363-72.

[21] Spronk HM, ten Cate H, van der Meijden PE. Differential roles of tissue factor and phosphatidylserine in activation of coagulation. Thrombosis research 2014;133 Suppl 1:S54-6.

[22] Morrissey JH. Tissue factor: a key molecule in hemostatic and nonhemostatic systems. International journal of hematology 2004;79:103-8.

[23] Moll G, Drzeniek N, Kamhieh-Milz J, Geissler S, Volk HD, Reinke P. MSC Therapies for COVID-19: Importance of Patient Coagulopathy, Thromboprophylaxis, Cell Product Quality and Mode of Delivery for Treatment Safety and Efficacy. Frontiers in immunology 2020;11:1091.

[24] Tang N, Li D, Wang X, Sun Z. Abnormal coagulation parameters are associated with poor prognosis in patients with novel coronavirus pneumonia. Journal of thrombosis and haemostasis : JTH 2020;18:844-7.

[25] Zhang Y, Xiao M, Zhang S, Xia P, Cao W, Jiang W, et al. Coagulopathy and Antiphospholipid Antibodies in Patients with Covid-19. The New England journal of medicine 2020;382:e38.

[26] Klok FA, Kruip M, van der Meer NJM, Arbous MS, Gommers D, Kant KM, et al. Incidence of thrombotic complications in critically ill ICU patients with COVID-19. Thrombosis research 2020;191:1457.

[27] François M, Copland IB, Yuan S, Romieu-Mourez R, Waller EK, Galipeau J. Cryopreserved mesenchymal stromal cells display impaired immunosuppressive properties as a result of heat-shock response and impaired interferon- $\gamma$ licensing. Cytotherapy 2012;14:147-52.

[28] Caplan H, Olson SD, Kumar A, George M, Prabhakara KS, Wenzel P, et al. Mesenchymal Stromal Cell Therapeutic Delivery: Translational Challenges to Clinical Application. Frontiers in immunology 2019;10:1645.

[29] Ocansey DKW, Pei B, Yan Y, Qian H, Zhang X, Xu W, et al. Improved therapeutics of modified mesenchymal stem cells: an update. Journal of translational medicine 2020;18:42.

[30] Mennan C, Garcia J, Roberts S, Hulme C, Wright K. A comprehensive characterisation of large-scale expanded human bone marrow and umbilical cord mesenchymal stem cells. Stem cell research \& therapy 2019;10:99.

[31] Giri J, Galipeau J. Mesenchymal stromal cell therapeutic potency is dependent upon viability, route of delivery, and immune match. Blood advances 2020;4:1987-97.

[32] Allen A, Vaninov N, Li M, Nguyen S, Singh M, Igo P, et al. Mesenchymal Stromal Cell Bioreactor for Ex Vivo Reprogramming of Human Immune Cells. Scientific reports 2020;10:10142.

[33] Trevisan BM, Porada CD, Atala A, Almeida-Porada G. Microfluidic devices for studying coagulation biology. Seminars in cell \& developmental biology 2020.

[34] Tilley D, Levit I, Samis JA. Development of a microplate coagulation assay for Factor $V$ in human plasma. Thrombosis journal 2011;9:11.

[35] Suidan GL, Singh PK, Patel-Hett S, Chen ZL, Volfson D, Yamamoto-Imoto H, et al. Abnormal clotting of the intrinsic/contact pathway in Alzheimer disease patients is related to cognitive ability. Blood advances 2018;2:954-63.

[36] Gleeson BM, Martin K, Ali MT, Kumar AH, Pillai MG, Kumar SP, et al. Bone Marrow-Derived Mesenchymal Stem Cells Have Innate Procoagulant Activity and Cause Microvascular Obstruction Following Intracoronary Delivery: Amelioration by Antithrombin Therapy. Stem cells (Dayton, Ohio) 2015;33:2726-37.

[37] Liao L, Shi B, Chang H, Su X, Zhang L, Bi C, et al. Heparin improves BMSC cell therapy: Anticoagulant treatment by heparin improves the safety and therapeutic effect of bone marrow-derived mesenchymal stem cell cytotherapy. Theranostics 2017;7:106-16. 
O’Rourke et al, Bioreactor Attenuates Clot Formation

[38] Coppin L, Najimi M, Bodart J, Rouchon MS, van der Smissen P, Eeckhoudt S, et al. Clinical Protocol to Prevent Thrombogenic Effect of Liver-Derived Mesenchymal Cells for Cell-Based Therapies. Cells 2019;8. [39] Cronin RE, Reilly RF. Unfractionated heparin for hemodialysis: still the best option. Seminars in dialysis 2010;23:510-5.

[40] Galipeau J, Sensébé L. Mesenchymal Stromal Cells: Clinical Challenges and Therapeutic Opportunities. Cell stem cell 2018;22:824-33.

[41] Moll G, Ankrum JA, Kamhieh-Milz J, Bieback K, Ringdén O, Volk HD, et al. Intravascular Mesenchymal Stromal/Stem Cell Therapy Product Diversification: Time for New Clinical Guidelines. Trends in molecular medicine 2019;25:149-63.

[42] Lalu MM, McIntyre L, Pugliese C, Fergusson D, Winston BW, Marshall JC, et al. Safety of cell therapy with mesenchymal stromal cells (SafeCell): a systematic review and meta-analysis of clinical trials. PloS one 2012;7:e47559.

[43] Yong KW, Choi JR, Wan Safwani WK. Biobanking of Human Mesenchymal Stem Cells: Future Strategy to Facilitate Clinical Applications. Advances in experimental medicine and biology 2016;951:99110.

[44] Kabat M, Bobkov I, Kumar S, Grumet M. Trends in mesenchymal stem cell clinical trials 2004-2018: Is efficacy optimal in a narrow dose range? Stem cells translational medicine 2020;9:17-27.

[45] Börger V, Weiss DJ, Anderson JD, Borràs FE, Bussolati B, Carter DRF, et al. ISEV and ISCT statement on EVs from MSCs and other cells: considerations for potential therapeutic agents to suppress COVID19. Cytotherapy 2020;22:482-5.

[46] Godoy JAP, Paiva RMA, Souza AM, Kondo AT, Kutner JM, Okamoto OK. Clinical Translation of Mesenchymal Stromal Cell Therapy for Graft Versus Host Disease. Frontiers in cell and developmental biology 2019;7:255.

[47] Elgaz S, Kuçi Z, Kuçi S, Bönig H, Bader P. Clinical Use of Mesenchymal Stromal Cells in the Treatment of Acute Graft-versus-Host Disease. Transfusion medicine and hemotherapy : offizielles Organ der Deutschen Gesellschaft fur Transfusionsmedizin und Immunhamatologie 2019;46:27-34.

[48] Horbett TA. Fibrinogen adsorption to biomaterials. Journal of biomedical materials research Part $A$ 2018;106:2777-88.

[49] Biran R, Pond D. Heparin coatings for improving blood compatibility of medical devices. Advanced drug delivery reviews 2017;112:12-23.

[50] Maul TM MM, Wearden PD. ECMO biocompatibility: surface coatings, anticoagulation, and coagulation monitoring. Extracorporeal Membrane Oxygenation-Advances in Therapy: InTechOpen2016.

[51] Nilsson B, Korsgren O, Lambris JD, Ekdahl KN. Can cells and biomaterials in therapeutic medicine be shielded from innate immune recognition? Trends in immunology 2010;31:32-8.

[52] Antebi B, Asher AM, Rodriguez LA, 2nd, Moore RK, Mohammadipoor A, Cancio LC. Cryopreserved mesenchymal stem cells regain functional potency following a 24-h acclimation period. Journal of translational medicine 2019;17:297.

[53] Moll G, Alm JJ, Davies LC, von Bahr L, Heldring N, Stenbeck-Funke L, et al. Do cryopreserved mesenchymal stromal cells display impaired immunomodulatory and therapeutic properties? Stem cells (Dayton, Ohio) 2014;32:2430-42.

[54] Szamosfalvi B, Yessayan L. Innovations in CKRT: individualized therapy with fewer complications. Nature reviews Nephrology 2020;16:560-1.

[55] Liang B, Chen J, Li T, Wu H, Yang W, Li Y, et al. Clinical remission of a critically ill COVID-19 patient treated by human umbilical cord mesenchymal stem cells: A case report. Medicine 2020;99:e21429. [56] Leng Z, Zhu R, Hou W, Feng Y, Yang Y, Han Q, et al. Transplantation of ACE2(-) Mesenchymal Stem Cells Improves the Outcome of Patients with COVID-19 Pneumonia. Aging and disease 2020;11:216-28. 
bioRxiv preprint doi: https://doi.org/10.1101/2020.11.20.391631; this version posted November 24, 2020. The copyright holder for this preprint (which was not certified by peer review) is the author/funder. All rights reserved. No reuse allowed without permission.

O’Rourke et al, Bioreactor Attenuates Clot Formation

583 [57] Limited M. U.S. FDA Advisory Committee Votes Nine to One in Favor of Remestemcel-L (Ryoncil ${ }^{\mathrm{TM}}$ ) 584 for Efficacy in Children With Steroid-Refractory Acute Graft Versus Host Disease. GlobeNewswire2020. 


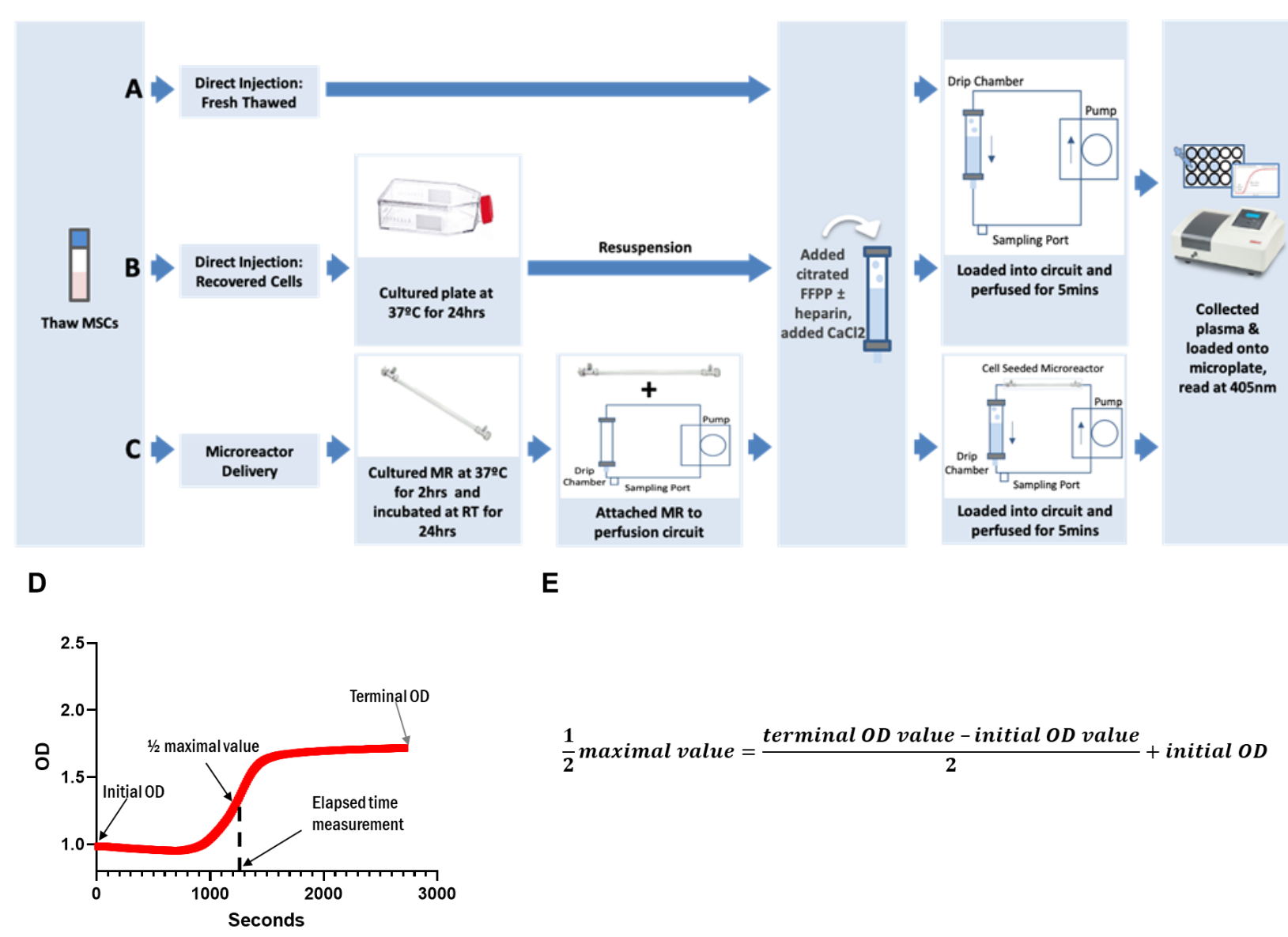

Figure 1. Clot Formation Assay Experimental Setup. MSCs were thawed from cryopreservation and were either (A) immediately combined into fresh-frozen pooled plasma-based (FFPP), perfused in the circuit and read, (B) cultured for 24 hours then resuspended into FFPP, perfused in the circuit and read, or (C) seeded into micro bioreactors (MR), allowed to attach to the hollow-fiber filters for 2 hours, room temperature incubated for 24 hours then attached to perfusion circuits loaded with FFPP, perfused and read. Perfusion of the MR circuits lasted 5 minutes before samples were collected and read on the spectrophotometer at $405 \mathrm{~nm}$ to assess fibrin formation. (D) Resulting spectrophotometric optical density (OD) readouts were graphed over time. (E) Formula used for the calculation of the clot formation time determined at the $1 / 2$ maximal value. 
A

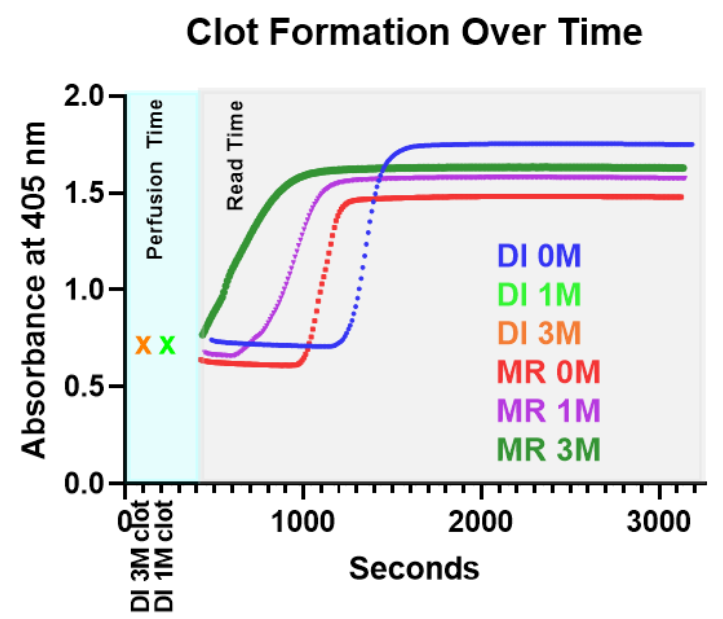

B

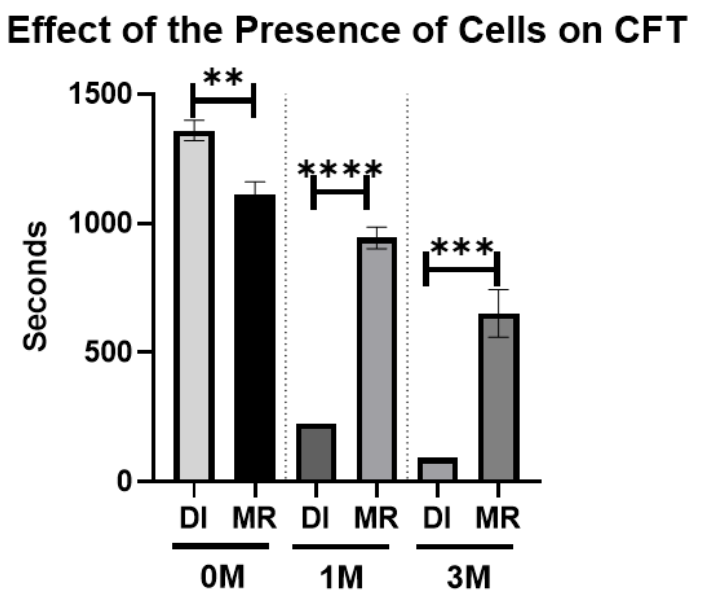

Figure 2. Increased MSC Exposure Shortens Clot Formation Time. 0, $1 \times 10^{6}$, or $3 \times 10^{6}$ viable MSCs were isolated for inclusion in each respective group. Freshly thawed MSCs were used in direct injection (DI) groups. MSCs used in MR groups were first incubated for 2 hours at $37^{\circ} \mathrm{C}$ followed by a 24 hour hold at room temperature prior to perfusion. After each groups' cells were prepared warmed fresh frozen pooled plasma was perfused through circuit for 5 minutes then subjected to spectrophotometric measurements. (A) Measurements of fibrin clot formation in plasma were made every 10 seconds over a 45-minute period (grey shaded region) following 5 minutes of perfusion (aqua shaded region). Groups which clotted during perfusion are designated with an ' $x$ ' at the time at which the clot was noted to be visibly obstructing perfusion. As clots formed, absorbance increased resulting in the designated curves. (B) Values for CFT were determined. Resulting values were graphed and analyzed with an unpaired student's t-test. $\mathrm{N}=3$ runs per group. ${ }^{* *}=p<0.005 ;{ }^{* * *}=p=0.0005 ;{ }^{* * * *}=p<0.0001$. Error bars represent \pm standard deviation. $\mathrm{DI}=$ direct injection 

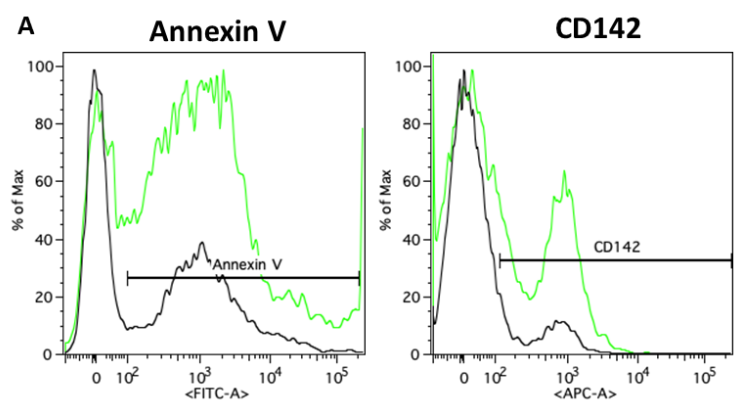

Fresh Thawed MSC

24hr Recovered MSC
B



Figure 3. The Effect of Cell Washing and Cell Recovery on CFT. $1 \times 10^{6}$ viable MSCs were isolated for inclusion in each respective group. Recovered cells were cultured for 24 hours and dissociated from the culture plate immediately prior to use. (A) Cells collected immediately after thaw and cells collected after recovery were subjected to staining and flow cytometry. Each curve represents the outcome of 3 pooled samples. (B) Direct injection groups were either thawed directly into plasma, washed with saline, or recovered with 24 hrs of culture at $37^{\circ} \mathrm{C}$. Microreactor groups were seeded with MSCs and either immediately used or allowed to attach for 2 hours at $37^{\circ} \mathrm{C}$ followed by a 24 hour hold at room temperature prior to perfusion. Values for CFT were determined and resulting values were graphed and analyzed with an unpaired student's t-test. $\mathrm{N}=3$ runs per group. ${ }^{* * * *}=p<0.0001$. Error bars represent \pm standard deviation. 
A

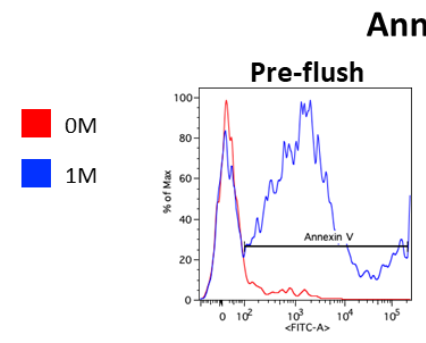

\section{Annexin V}
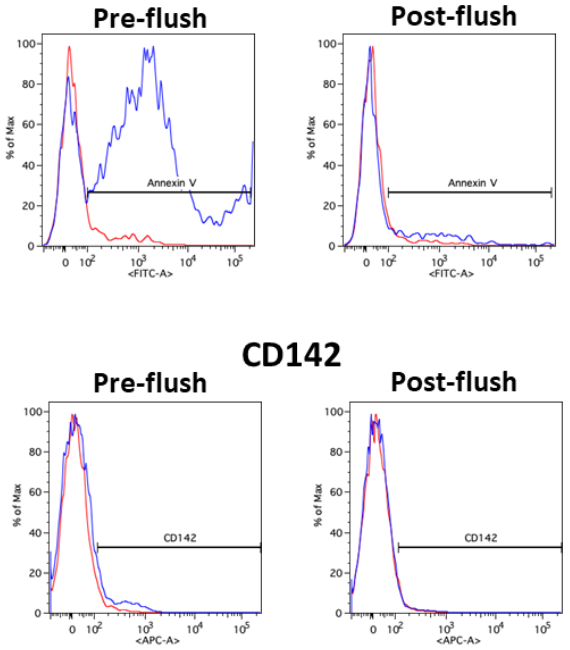

CD142



B

\section{Effect of Flushing Microreactors on CFT}

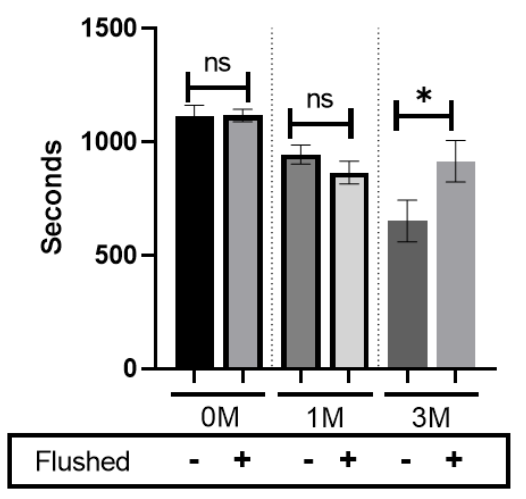

Figure 4. Flushing of Soluble Factors Affects CFT. Microreactors were seeded with MSCs and allowed to attach for 2 hours at $37^{\circ} \mathrm{C}$ followed by a 24 hour hold at room temperature. (A) Samples taken from the extracapillary space of microreactors with either 0 or $1 \times 10^{6} \mathrm{MSCs}$, both pre- and post-flushing of the device, were subjected to staining and flow cytometry for known pro-coagulation markers phosphatidylserine (Annexin V) and tissue factor (CD142). Each curve represents the outcome of 3 pooled samples. (B) Warmed fresh frozen pooled plasma was perfused through circuits with either 0,1 or $3 \times 10^{6}$ viable MSCs (with and without a flushing procedure) for 5 minutes then subjected to spectrophotometric measurements. Measurements of fibrin clot formation in plasma were then made every 10 seconds over a 45-minute period. Values for CFT were determined and values were graphed and analyzed with an unpaired student's t-test. $\mathrm{N} \geq 2$ runs per group. ${ }^{*}=\mathrm{p}<0.05$. Error bars represent \pm standard deviation. 


\section{Effect of Heparin (1.5 U/mL) on CFT}

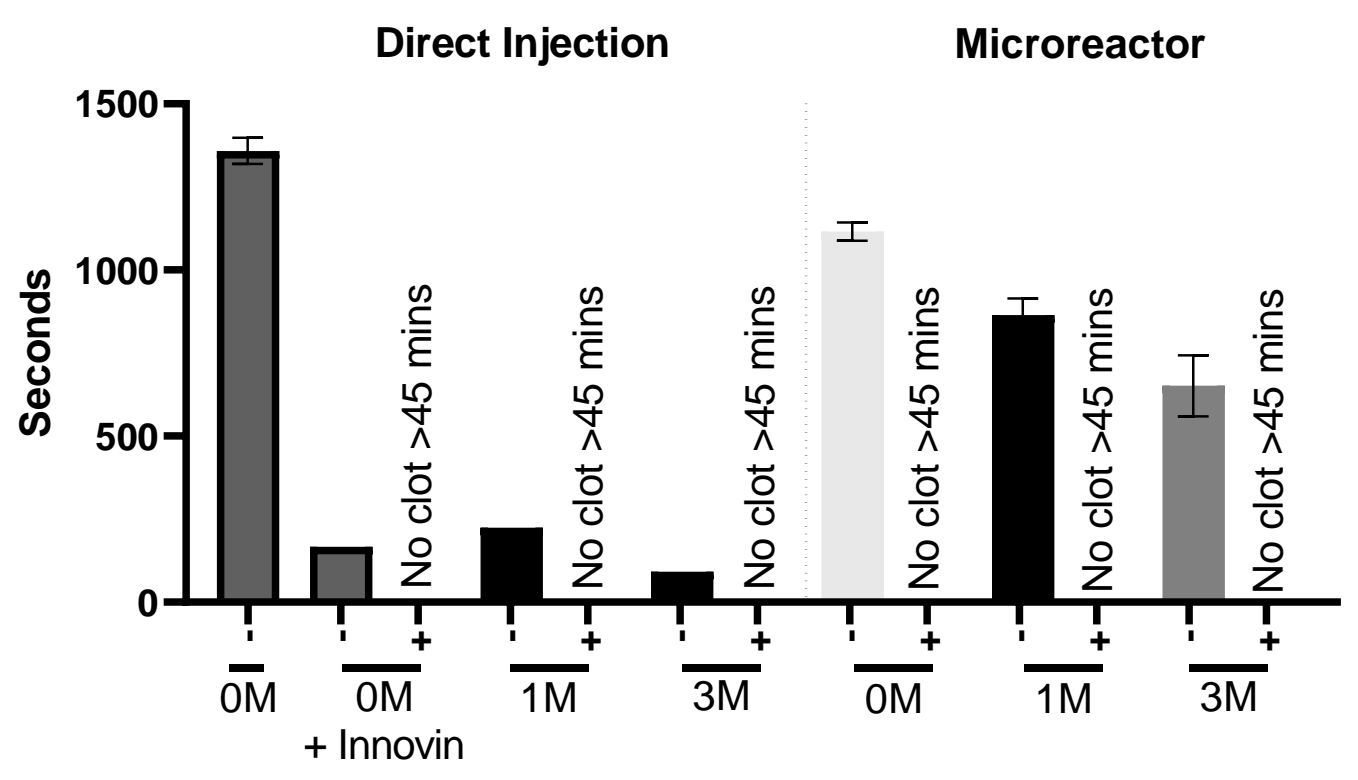

Figure 5. Effect of Heparin on CFT. $0,1 \times 10^{6}$, or $3 \times 10^{6}(\mathrm{OM}, 1 \mathrm{M}, 3 \mathrm{M})$ viable MSCs were isolated for inclusion in each respective group. MSCs for direct injection groups were thawed directly into plasma then used, while microreactor groups were seeded with MSCs and allowed to attach for 2 hours at $37^{\circ} \mathrm{C}$ followed by a 24 hour hold at room temperature prior to perfusion. Innovin (thromboplastin) was added to the $0 \mathrm{M}$ group as a positive control. After each groups' cells were prepared, warmed FFPP was perfused through circuit for 5 minutes then subjected to spectrophotometric measurements. Measurements of fibrin clot formation in plasma were made every 10 seconds over a 45-minute period. Values for CFT were determined and resulting values were graphed and analyzed with an unpaired student's t-test. Samples which showed no increase in absorbance through the course of the experiment were designated to have not clotted. $\mathrm{N} \geq 2$ runs per group. $*=p<0.05$. Error bars represent \pm standard deviation. 
A

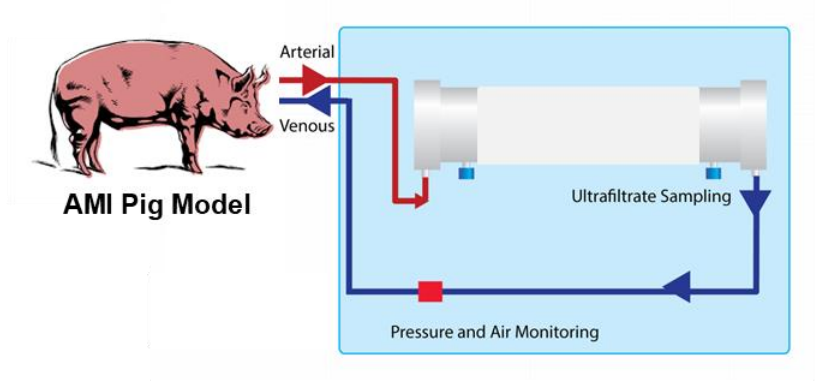

C

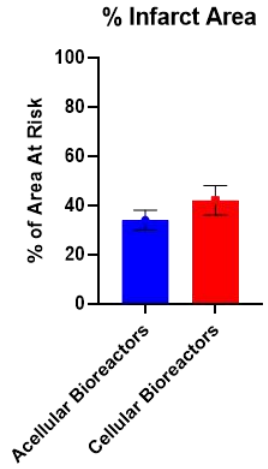

B

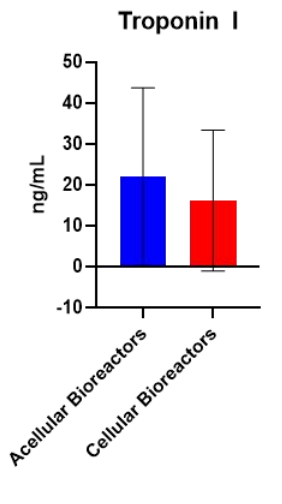

D



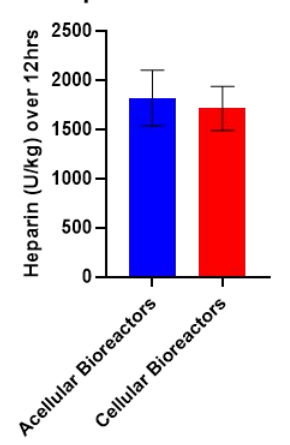

Figure 6. Pig AMI Model Perfusion. (A) Pigs were sedated, occluded of their left anterior descending coronary artery, re-perfused for 1 hour, and administered the designated bioreactor (acellular or cellular) for 12 hours of perfusion. (B,C) Comparative measurements of induced stress were done through serum sampling of Troponin I levels at the 12 hour mark, as well as morphological analysis of \% infarct area at sacrifice. (D,E) Heparin was administered over the course of 12 hours to maintain an ACT of approximately 300 seconds. $\mathrm{N}=4$ pigs per group. Error bars represent \pm standard deviation. 